\title{
Effect of Repeated Oral Health Education on the Oral Health of Preschool Children
}

\author{
Jee-Won Lee and Ha-Na Choi $^{\dagger}$ \\ Department of Dental Hygiene, Jeonju Kijeon College, Jeonju 54989, Korea
}

This study aimed to investigate the effect of repeated oral health education on the oral health of preschool children. This study classified the control group of children of $5 \sim 6$ years of age where the oral health education was conducted one time, and the experiment group where the oral health education was conducted 4 times. The oral health conditions of each group and their risk of developing dental caries were also assessed. No significant difference was observed in the participants in terms of general characteristics, oral health conditions, risk of developing dental caries, and oral health behavior after oral health education. The two groups did not show a significant difference $(p<0.001)$ in dental plaque index before and after oral health education, and showed a significant difference $(p<0.001)$ according to the number of education sessions. Before oral health education, the participants in both groups were brushing their teeth incorrectly. However, after the third session, $56.7 \%$ of the participants in the experimental group and $9.4 \%$ in the control group used the correct method of tooth brushing. A significant difference was observed between the two groups $(p<0.05)$. In addition, when the tooth-brushing time was compared, the two groups showed a significant difference $(p<0.001)$, and a significant difference was observed in terms of the number of education sessions $(p<0.05)$. In summary, repeated oral health education had significant influences on the dental plaque index, tooth-brushing method, brushing occlusal surfaces, and tooth-brushing time. Therefore, when oral health education is carried out, repeated oral health education in children is more effective than one-time oral health education as reflected in the changes in dental plaque index and oral health behavior. Moreover, oral health education is considered effective in changing the oral health behavior of children when conducted at least 3 times.

Key Words: Dental caries activity tests, Dental plaque index, Oral health education, Preschool children, Toothbrushing

\section{Introduction}

Children develop health awareness and patterns of health behaviors during the preschool period, which is the basis for the establishment of a lifetime of good health ${ }^{1)}$. Dental caries that develop during the preschool period can pose serious physical, psychological, and social danger to a child if not promptly and appropriately treated, causing significant financial burden, and lead to systemic infections $^{2)}$. This multifactorial disorder that is caused by lifestyle factors, which is similar to other chronic diseases $^{3,4)}$, can be prevented by performing health behaviors, such as regular tooth brushing, consumption of a healthy diet, and constant dental examinations.
Oral health education helps children develop oral care habits and promotes a healthy lifestyle and is important in the prevention of oral diseases, such as dental caries and periodontal diseases. Furthermore, it aims to improve and maintain oral health and promote changes in the attitude, level of knowledge, and behavior related to oral health management. Thus, the contents and methods of oral health education must be tailored based on individual characteristics ${ }^{5)}$. Although preschool educational institutions are responsible for oral health care education, its implementation is still unsatisfactory ${ }^{6,7}$. Korea has implemented oral health care projects for pregnant women and infants in accordance with Article 16 of the Oral Health Care Act. Article 12 of the Oral Health Care Act also 
requires schools to implement oral health education, oral health examinations, group tooth brushing, fluoride rinsing, and regular oral health care $^{8}$. However, oral health education for preschool children has not been vigorously implemented, and because of the limited period or number of education sessions, significant educational effects are difficult to achieve ${ }^{9)}$. Lee et $\mathrm{al}^{10)}$. have reported that educating individuals about tooth brushing only once is not sufficient in improving oral health. In addition, the effects of tooth-brushing education regressed at $4 \sim 6$ weeks after the education. Therefore, changes in oral health behaviors as a result of short-term education or one-time education are not easily obtained.

Previous studies, ${ }^{6,11-14)}$ have shown that a group subjected to one or more sessions of oral health education effectively managed their oral health compared to a group that did not receive oral health education at all. Thus, oral health education is an important method in managing the oral health of children. However, differences in the effects on the oral health of children as a result of one-time and repeated oral health education have not been investigated. Therefore, whether oral health care effects can be achieved through one-time oral health education only or repeated education would produce better effects should be evaluated. If repeated education is more effective in promoting oral health care, then the number of education sessions must be repeated to produce maximal effects on the oral health care of children.

Previous studies have investigated the effects of oral health education methods and oral health care programs ${ }^{15,16)}$ and differences in the level of oral health care according to the type of tooth-brushing environment ${ }^{17)}$. In addition, the effects of oral health education were assessed ${ }^{11-13)}$. However, the period or the number of education sessions was limited in these studies. Others have investigated the effects of repeated tooth-brushing education $^{6,14)}$. However, no significant difference was observed in the effects of one-time education and repeated education. Another study has conducted a risk assessment for dental care based on bacterial testing of Streptococcus mutans and lactobacilli using saliva ${ }^{6,14)}$. Results showed that dental caries are worsened by the actions of multiple microbes within the oral cavity rather than by one specific type of causative bacteria. The total amount of organic acid released by all microbes within the oral cavity can be used as an important marker for predicting dental caries $^{18,19)}$. The Cariview ${ }^{\mathrm{TM}}$ (Huneth, Seoul, Korea) test measures the amount of organic acid produced by all microbes that induce dental caries within the oral cavity to assess oral health conditions. It accurately predicts dental caries compared to other existing tests that use saliva. Thus, the present study aimed to investigate the effects of one-time and repeated tooth-brushing education, which is one of the important parts of oral health education ${ }^{20)}$, on the oral health conditions and behavior of children aged $5 \sim 6$ years who are enrolled in childcare institutions. In addition, the rationale for the methods of oral health education for children and the roles of dental hygienists were established.

\section{Materials and Methods}

\section{Subjects}

This study was conducted after the approval from the Institutional Review Board of Wonkwang University (IRB no. WKIRB-201505-SB-020). Children who were attending kindergarten or daycare centers in Jeonju, Jeollabuk-do, were included in the present study. Eighty-three children and their parents consented to participate. Of these children, those who satisfied the inclusion and exclusion criteria were finally included. The inclusion criteria included an age of $5 \sim 6$ years and the ability to communicate. Children with systemic disorders were excluded. Of the children who consented to participate, 68 satisfied the inclusion criteria. Thirty-three of these children were assigned to the control group and 35 children to the experimental group. Six children were withdrawn because of absences and shifting of home during the research period. Ultimately, a total of 62 children were considered as the final participants (40 boys and 22 girls), 32 of whom were in the control group and 30 of whom were in the experimental group. No significant differences were observed between the two groups in terms of sex and age distributions. 


\section{Methods}

\section{1) Study design}

This study used a nonequivalent control group design. The study purpose and method were explained to the children and their legal guardians, and a written consent was provided. Children were classified into two different groups to investigate the effects of repeated oral health education as shown in Fig. 1. Both groups were subjected to tooth-brushing education on the first education session. After the first oral health education session, the control group had a total of four oral health examinations at a 2-week interval. The experimental group attended four sessions of tooth-brushing education every 2 weeks and had an oral health examination on the second week of education. The oral health conditions of the control group subjected to one-time education and the experimental group subjected to repeated education consisting of four sessions were compared.

\section{2) Oral health education}

Oral health education was conducted by two dental hygienists, including author, and mainly focused on tooth brushing. Oral health education materials that were provided by the Korean Academy of Oral Health (2006) were used in making the education contents, and we consulted a dentist, two dental hygienists, and two kindergarten teachers to revise and improve the contents. The participating children were taught the Fone's method at a kindergarten classroom by the researcher. Children who do not know how to properly brush their teeth easily learned the Fone's method and later switched from using the rotation method to the Fone's method ${ }^{21)}$. After teaching the Fone's method, the researcher observed how the children brushed their teeth and corrected them when necessary.

\section{3) Assessment instrument}

Oral health conditions were evaluated through dental



Fig. 1. Flow diagram of study design. 
caries test, dental plaque test, and dental caries risk assessment. A dentist who did not participate in the education assessed decayed teeth (dt), filled teeth (ft), and decayed and filled teeth (dft). A dental hygienist who did not participate in the education assessed dental plaques and the risk of developing dental caries. The O'Leary index was measured during the dental plaque test. Of the various methods used for the risk assessment of dental carries, the Cariview ${ }^{\mathrm{TM}}$ test was used in this study. Based on the Cariview ${ }^{\mathrm{TM}}$ test, the total amount of organic acids released by the microbes inside the oral cavity is measured based on color changes that occur in accordance with $\mathrm{pH}$ changes. A sterilized cotton swab was rubbed against the tooth surface to collect a dental plaque sample, which was then placed in a tube and incubated at $37^{\circ} \mathrm{C}$ for 48 hours. When incubation was completed, 10 drops of the diagnostic agent were added, and induced color changes were analyzed using an optical analyzer. Participants were then classified into the low-risk, medium-risk, and high-risk groups based on the Cariview ${ }^{\mathrm{TM}}$ test scores of $0.0 \sim 40.0,41.0 \sim 70.0$, and 71.0 100.0, respectively.

For the oral health behavior assessment, brushing methods and time and whether or not the children at each childcare institution brushed the occlusal surfaces were investigated. Children and their teachers were interviewed, and the former were also monitored.

\section{Statistical analysis}

Collected data were analyzed using IBM SPSS Statistics ver. 21.0 (IBM Co., Armonk, NY, USA). A chi-square test and independent t-test were conducted to obtain differences between the two groups in terms of sex,

Table 1. General Characteristics of the Subjects

\begin{tabular}{ccccc}
\hline Characteristic & $\begin{array}{c}\text { Control } \\
\text { group }(\mathrm{n}=32)\end{array}$ & $\begin{array}{c}\text { Experimental } \\
\text { group }(\mathrm{n}=30)\end{array}$ & $\chi^{2}$ & $\mathrm{p}$ \\
\hline Gender & & & 0.518 & 0.325 \\
Male & $22(68.8)$ & $18(60.0)$ & & \\
Female & $10(31.2)$ & $12(40.0)$ & & \\
Age (y) & & & 0.061 & 0.503 \\
5 & $15(46.9)$ & $15(50.0)$ & & \\
6 & $17(53.1)$ & $15(50.0)$ & & \\
\hline
\end{tabular}

Values are presented as $\mathrm{n}(\%)$.

$\mathrm{p}<0.05$ by chi-square test. age, oral health conditions, oral heath behavior, and Cariview scores before oral health education. A paired t-test was carried out to obtain the differences between the two groups in terms of the level of dental caries before and after oral health education. A repeated measures ANOVA was used to compare the dental plaque index, risk of developing dental caries, and tooth-brushing time according to the type of oral health education conducted for each group. A chi-square test was carried out to evaluate tooth-brushing methods and whether or not the participants from each group brushed the occlusal surfaces after tooth-brushing education.

\section{Results}

\section{General characteristics and oral health conditions of the participants}

Table 1 shows the gender distribution of the control group (boys, 68.8\%; girls, 31.2\%) and the experimental group (boys, 60.0\%; girls, 40.0\%). No significant difference was observed between the two groups in terms of gender distribution. About 53.1\% and $46.9 \%$ of the participants in the control group were composed of children aged 6 years and 5 years, respectively, and $50.0 \%$ of the participants in the experimental group were composed of children aged 6 years. No significant difference was observed between the groups in terms of age distribution ( $\mathrm{p}>0.05)$.

No significant differences were found between the two groups in terms of the level of dental caries, dental plaque index, risk of developing dental caries, and tooth-brushing time as shown in Table 2 ( $\mathrm{p}>0.05)$. Thus, education was conducted under identical conditions.

\section{Oral health conditions after oral health education}

\section{1) Effects of oral health education on the rate of dental caries}

No significant differences were observed in the control group in terms of the rate of $\mathrm{dt}$ and $\mathrm{ft}$ before and after education as shown in Table 3 ( $\mathrm{p}>0.05)$. 
Table 2. Oral Health Status and Oral Health Behavior of the Subjects

\begin{tabular}{lccrc}
\hline \multicolumn{1}{c}{ Variable } & Control group $(\mathrm{n}=32)$ & Experimental group $(\mathrm{n}=30)$ & $\mathrm{t}$ & $\mathrm{p}$ \\
\hline $\mathrm{dt}$ & $0.47 \pm 1.14$ & $0.63 \pm 1.03$ & 0.596 & 0.554 \\
$\mathrm{ft}$ & $2.14 \pm 1.43$ & $1.10 \pm 0.88$ & 0.490 & 0.626 \\
$\mathrm{dft}$ & $1.34 \pm 1.75$ & $1.73 \pm 2.20$ & 0.774 & 0.442 \\
O'Leary index $^{\mathrm{a}}$ & $79.56 \pm 12.47$ & $80.46 \pm 14.79$ & 0.260 & 0.795 \\
Cariview score $^{\mathrm{b}}$ & $58.74 \pm 16.58$ & $57.68 \pm 15.93$ & -0.256 & 0.798 \\
Tooth brushing time $^{\mathrm{c}}$ & $37.66 \pm 12.55$ & $38.50 \pm 9.37$ & 0.298 & 0.767 \\
\hline
\end{tabular}

Values are presented as mean \pm standard deviation.

$\mathrm{dt}$ : decay teeth, ft: filling teeth, dft: decayed and filled teeth.

$\mathrm{p}<0.05$ by independent samples t-test.

${ }^{\mathrm{a}}$ Plaque control record, ${ }^{\mathrm{b}}$ comprehensive analysis of cariogenic potential of whole bacteria, ${ }^{\mathrm{c}}$ seconds.

Table 3. The Difference of $\mathrm{dt}$, $\mathrm{ft}$ Index between Two Groups according to the Toothbrushing Education

\begin{tabular}{|c|c|c|c|c|}
\hline Variable & Baseline & 2 weeks after 4 th education & $\mathrm{t}$ & $\mathrm{p}$ \\
\hline \multicolumn{5}{|l|}{$\mathrm{dt}$} \\
\hline Control group $(n=32)$ & $0.47 \pm 1.14$ & $0.47 \pm 1.14$ & & \\
\hline Experimental group $(n=30)$ & $0.63 \pm 1.03$ & $0.67 \pm 1.09$ & -0.571 & 0.573 \\
\hline \multicolumn{5}{|l|}{$\mathrm{ft}$} \\
\hline Control group $(n=32)$ & $0.88 \pm 1.43$ & $0.88 \pm 1.43$ & & \\
\hline Experimental group $(n=30)$ & $1.10 \pm 2.14$ & $1.13 \pm 2.13$ & -1.000 & 0.326 \\
\hline
\end{tabular}

Values are presented as mean \pm standard deviation.

$\mathrm{dt}$ : decay teeth, ft: filling teeth.

$\mathrm{p}<0.05$ by paired t-test.

Table 4. The Difference of O'Leary Index between Two Groups according to the Toothbrushing Education

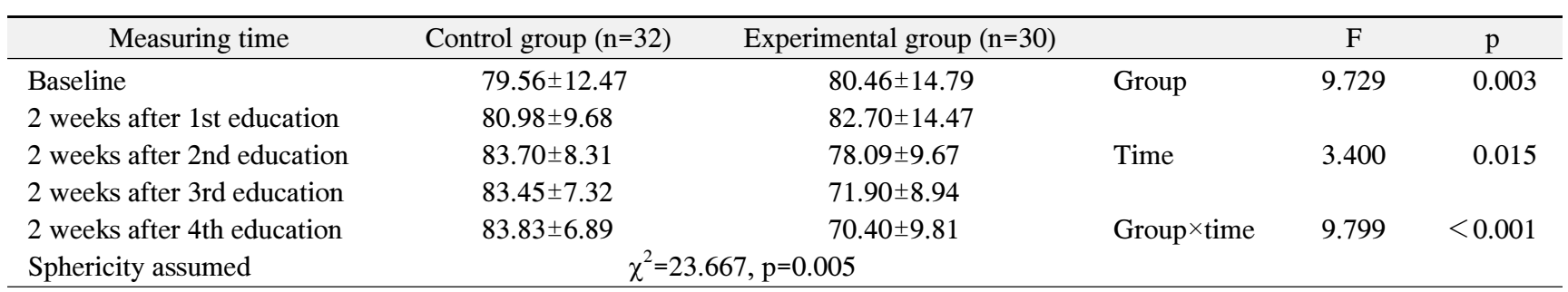

Values are presented as mean \pm standard deviation.

$\mathrm{p}<0.05$ by repeated measures ANOVA.

\section{2) Effects of oral health education on dental plaque conditions}

To investigate if repeated tooth-brushing education promoted significant changes in the dental plaque index, a repeated measures ANOVA was used. The assumption of sphericity was satisfied $(\mathrm{p} \geq 0.05)$. As shown in Table 4 , significant differences were observed between the two groups in terms of dental plaque index $(\mathrm{p}<0.005)$. The dental plaque index of the experimental group was lower than that of the control group. Significant differences were observed in the dental plaque index based on the number of education sessions held within $(\mathrm{p}<0.05)$ and between ( $p<0.001)$ the groups.

\section{3) Effects of oral health education on the risk of dental caries}

A repeated measures ANOVA was used to investigate if repeated oral health education promoted significant changes in the risk of developing dental caries. The assumption of sphericity was satisfied $(\mathrm{p} \geq 0.05)$. As 
shown in Table 5, a significant difference was observed between the control and experimental groups in terms of the risk of developing dental caries $(p<0.05)$. No significant difference was observed between the two groups in terms of the risk of developing dental caries based on the number of education sessions held within $(p>0.05)$ and between $(p>0.05)$ the groups.

\section{Effects of oral health education on oral health behaviors}

\section{1) Effects of oral health education on the brushing methods used}

The tooth-brushing methods used by the two groups were compared, and results showed that both groups did not use the Fone's method as shown in Table 6. After the third education session, significant differences were observed between the two groups in terms of the types of brushing method used $(\mathrm{p}<0.05)$. About $9.4 \%$ of the participants in the control group and $56.7 \%$ of those in the experimental groups used the Fone's method. After the fourth education session, significant differences were observed between the two groups in terms of the type of brushing method used ( $p<0.05$ ). In addition, $6.2 \%$ of the participants in the control group and $73.3 \%$ of those in the experimental group used proper brushing methods.

2) Effects of oral health education on the number of patients who brush occlusal surfaces

As shown in Table 7, no significant difference was

Table 5. The Difference of Cariview ${ }^{\mathrm{TM}}$ Test Score between Two Groups according to the Toothbrushing Education

\begin{tabular}{|c|c|c|c|c|c|}
\hline Measuring time & Control group $(n=32)$ & Experimental group $(n=30)$ & & $\mathrm{F}$ & $\mathrm{p}$ \\
\hline Baseline & $58.74 \pm 16.58$ & $57.68 \pm 15.93$ & Group & 4.045 & 0.049 \\
\hline 2 weeks after 1 st education & $62.12 \pm 10.59$ & $56.14 \pm 15.18$ & & & \\
\hline 2 weeks after 2 nd education & $61.30 \pm 14.35$ & $59.01 \pm 18.72$ & Time & 0.640 & 0.634 \\
\hline 2 weeks after 3 rd education & $57.54 \pm 12.26$ & $55.52 \pm 14.24$ & & & \\
\hline 2 weeks after 4 th education & $62.48 \pm 9.22$ & $53.55 \pm 13.81$ & Group $\times$ time & 0.957 & 0.432 \\
\hline Sphericity assumed & \multicolumn{2}{|c|}{$\chi^{2}=11.948, p=0.216$} & & & \\
\hline
\end{tabular}

Values are presented as mean \pm standard deviation.

$\mathrm{p}<0.05$ by repeated measures ANOVA.

Table 6. The Difference of Toothbrushing Method between Two Groups according to the Toothbrushing Education

\begin{tabular}{lccc}
\hline \multicolumn{1}{c}{ Toothbrushing method } & Control group $(\mathrm{n}=32)$ & Experimental group $(\mathrm{n}=30)$ & $\chi^{2}$ \\
\hline Baseline & $0(0.0)$ & $0(0.0)$ & \\
$\quad$ Fones'method & $32(100.0)$ & $30(100.0)$ & 2.119 \\
$\quad$ The other method & $13(40.6)$ & $7(23.3)$ & 0.146 \\
2 weeks after 1st education & $19(59.4)$ & $23(76.7)$ & 1.245 \\
$\quad$ Fones'method & $4(12.5)$ & $7(23.3)$ & 0.264 \\
$\quad$ The other method & $28(87.5)$ & $23(76.7)$ & 15.847 \\
2 weeks after 2nd education & $3(9.4)$ & $17(56.7)$ & $<0.001$ \\
$\quad$ Fones'method & $29(90.6)$ & $13(43.3)$ & \\
$\quad$ The other method & $2(6.2)$ & $22(73.3)$ & 8.370 \\
2 weeks after 3rd education & $30(93.8)$ & $8(26.7)$ & $<0.001$ \\
$\quad$ Fones'method & & & \\
$\quad$ The other method & & & \\
2 weeks after 4th education & Fones'method & & \\
$\quad$ The other method & & & \\
\hline
\end{tabular}

Values are presented as $\mathrm{n}(\%)$.

$\mathrm{p}<0.05$ by chi-square test. 
observed between the two groups in terms of the number of patients who brush occlusal surfaces $(\mathrm{p}>0.05)$.

\section{3) Effects of oral health education on the tooth-brushing time of the participants}

To investigate if repeated oral health education promoted significant changes in the brushing time of the participants, a repeated measures ANOVA was used. The assumption of sphericity was satisfied ( $p \geq 0.05$ ). Significant differences were observed between the two groups in terms of the brushing time $(\mathrm{p}<0.05)$ and elapsed time $(\mathrm{p}$ $<0.05$ ), indicating that the control group spent more time on brushing. No significant difference in the elapsed time was found between the two groups ( $p>0.05)$ (Table 8).

\section{Discussion}

In this study, children were classified into the control and experimental groups and subjected to tooth-brushing education to investigate the effects of repeated oral health education on the oral health of children. The control group was subjected to only one session of tooth-brushing education, followed by a total of four oral health examinations every 2 weeks. The experimental group was subjected to a total of four tooth-brushing education sessions at a 2-week interval and an oral health examination a week after each session. The oral health conditions of the group that received education only once and the group that attended four sessions of repeated education were compared.

Table 7. The Difference of Occlusal Brushing between Two Groups according to the Toothbrushing Education

\begin{tabular}{|c|c|c|c|c|}
\hline Occlusal brushing & Control group $(n=32)$ & Experimental group $(n=30)$ & $\chi^{2}$ & $\mathrm{p}$ \\
\hline Baseline & & & 2.349 & 0.185 \\
\hline Yes & $24(75.0)$ & $27(90.0)$ & & \\
\hline No & $8(25.0)$ & $3(10.0)$ & & \\
\hline 2 weeks after 1 st education & & & 1.727 & 0.355 \\
\hline Yes & $28(87.5)$ & $29(96.7)$ & & \\
\hline No & $4(12.5)$ & $1(3.3)$ & & \\
\hline 2 weeks after 2 nd education & & & 0.938 & 1.000 \\
\hline Yes & $31(96.9)$ & $30(100.0)$ & & \\
\hline No & $1(3.1)$ & $0(0.0)$ & & \\
\hline 2 weeks after 3 rd education & & & 5.016 & 0.053 \\
\hline Yes & $27(84.4)$ & $30(100.0)$ & & \\
\hline No & $5(15.6)$ & $0(0.0)$ & & \\
\hline 2 weeks after 4 th education & & & 0.281 & 1.000 \\
\hline Yes & $30(93.8)$ & $29(96.7)$ & & \\
\hline No & $2(6.3)$ & $1(3.3)$ & & \\
\hline
\end{tabular}

Values are presented as $\mathrm{n}(\%)$.

$\mathrm{p}<0.05$ by Fisher's exact test.

Table 8. The Difference of Toothbrushing Time between Two Groups according to the Toothbrushing Education (Unit: Second)

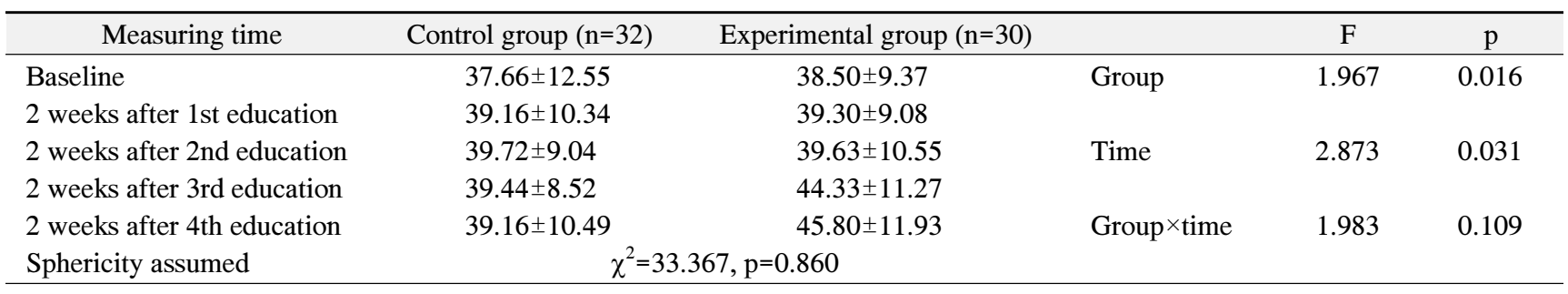

Values are presented as mean \pm standard deviation.

$\mathrm{p}<0.05$ by repeated measures ANOVA. 
After tooth-brushing education, a significant difference was observed between the control and experimental groups in terms of the dental plaque index which is in accordance with the number of education sessions. The dental plaque index of the experimental group was lower compared to that of the control group. After the first education session, no significant difference was observed between the two groups in terms of the dental plaque index. After the second education session, significant differences were observed between the two groups in terms of the dental plaque index $83.70 \pm 8.31$ for the control group and 78.09 \pm 9.67 for the experimental group). While no significant difference in the dental plaque index was found at each measurement in the control group, the dental plaque index significantly decreased by $6.20 \pm 15.65$ from 78.09 \pm 9.67 following the second session to $71.90 \pm 8.94$ after the third session in the experimental group. At this time point, the dental plaque index significantly decreased. Our results differed from those of You et al. ${ }^{22)}$ in which the dental plaque index significantly decreased from $3.63 \pm 0.72$ to $3.29 \pm 0.70$ after only one session of tooth-brushing education. In their study, improvements in brushing methods were observed after the second education session, and the dental plaque index also decreased after the first and second sessions, which were contrary to our results. Furthermore, our results differed from those of Song ${ }^{6}$ who investigated the effects of oral health education on children who are in kindergarten. In their study, no significant difference was observed between the control and experimental groups in terms of the dental plaque index before, immediately after, and at 4 and 28 weeks after education. Although Song ${ }^{6}$ also conducted repeated oral health education, the method used was based on passed on knowledge. On the other hand, a one-on-one tooth-brushing education was conducted using disclosing agents after the group tooth-brushing education, and proper tooth-brushing methods were taught to the children. By providing repeated education that focused on tooth brushing, the dental plaque index of the experimental group significantly decreased. Kang et al. ${ }^{14)}$ have also reported a significant decrease in the dental plaque index after oral health education as a result of repeated tooth-brushing education in which the brushing methods used by the children were monitored and corrected. Although the presence of dental plaques is an important factor that influences oral diseases, such as dental caries and periodontal diseases $^{21)}$, the initial dental plaque index of both groups had approximately 80 points, indicating that the dental plaques of children are poorly managed. According to Eom et al. $^{23)}$ tooth-brushing education should be conducted repeatedly and must include dental plaque management that is tailored based on the individual's oral characteristics and ability to manage oral health. To obtain certain levels of ability to manage dental plaques, at least three to four sessions of repeated education should be conducted. Choi et al. ${ }^{24)}$ have subjected elementary school students to repeated tooth-brushing education and reported that the number of students who use proper brushing techniques increased after three education sessions. In this study, changes in the dental plaque index were observed after two sessions, and the most significant reduction in the dental plaque index was observed after the third session. Therefore, repeated tooth-brushing education (three sessions) may be effective for dental plaque management.

The Cariview scores of the control and experimental group after the fourth session, which indicate the risk of dental caries, were 62.58 \pm 9.22 and 53.55 \pm 13.81 , respectively. A significant difference was observed between the two groups in terms of the scores. Kang et al. ${ }^{14)}$ have reported a significant decrease in the level of $S$. mutans but not lactobacilli in the experimental group that received tooth-brushing education on salivary testing that was conducted to assess the levels of caries activity. The method used for the risk assessment of dental caries in this study can highly predict dental caries compared to the existing methods that use saliva because it can assess the overall oral health based on the amount of organic acids produced by all microbes within the oral cavity, and the characteristics of the biofilms are maintained since dental plaques are directly collected and cultured ${ }^{18,19)}$. Therefore, contrary to the study by Kang et al. ${ }^{14)}$ in which changes in certain microbes in the saliva are examined after tooth-brushing education, our study assessed the amount of organic acids released by the microbes after the dental 
plaques are collected. The bacteria levels changed over time because the amount of dental plaques is reduced through tooth brushing.

This study investigated the effects of one-time and repeated tooth brushing education on the oral health of children, and significant changes were observed between the two groups in terms of dental plaque, risk of developing dental caries, and brushing methods and time. Differences in the dental plaque index were observed after the second session, and the most significant reduction in dental plaque index was observed after the third session. Differences in the dental plaque were still observed after the fourth session, and the number of children who were able to use proper brushing techniques significantly increased after the third session. Based on these results, repeated tooth-brushing education can efficiently promote the maintenance of children's oral health compared to one-time education. At least three sessions of oral health education are necessary to effectively manage dental plaques and improve the oral health of children, and more than four sessions of health education should be conducted considering the risk of developing dental caries.

Because this study involved children who are enrolled in kindergarten and daycare centers within a particular region, our results cannot be generalized to all children. Previous studies focused on the effects of one-time and repeated oral health education. However, the present study did not only asses the effects of one-time and repeated tooth-brushing education but also investigated the number of education sessions that is needed to obtain significant results.

\section{References}

1. Kim YH, Kwon BS, Yang YO, et al.: Child health nursing. Yangseowon, Seoul, pp.17-18, 2009.

2. Oral Health in America: a report of the surgeon general. J Calif Dent Assoc 28: 685-695, 2000.

3. Schroth RJ, Harrison RL, Moffatt ME: Oral health of indigenous children and the influence of early childhood caries on childhood health and well-being. Pediatr Clin North Am 56: 1481-1499, 2009.

4. Featherstone JD: The caries balance: contributing factors and early detection. J Calif Dent Assoc 31: 129-133, 2003.

5. Han JH: Effects of oral health education using play-for the kindergarten children. J Dent Hyg Sci 5: 57-62, 2005.

6. Song BS: The effect of oral health education on oral health in kindergarten children. Taehan Kanho Hakhoe Chi 34: 132-140, 2004.

7. Ahn YS, Kim ES, Lim DS, Jung SH, Kim MJ: A study on preschool teachers' oral health knowledge and behaviors in Sung-nam city. J Dent Hyg Sci 1: 13-21, 2001.

8. Ministry of Health and Welfare: Dental health act, Act No. 13319, Enforcement 2012 Oct. Ministry of Health and Welfare, Sejong, 2012.

9. Lee KH, Kim JY, Song JH, Kim YH, Im KU, Jeong SY: Present state and prospect of public dental health service for children and adolescents in Korea. J Korean Acad Pediatr Dent 35: 578-588, 2008.

10. Lee SS, Paik DI, Kim JB: A study on the effects of the toothbrushing instruction methods in dental health education. J Korean Acad Oral Health 14: 233-242, 1990.

11. Jeon HJ, Song KB, Lee SK: The improvement of knowledge and management capability of oral health according to the oral health education system for elementary school students. J Korean Soc Sch Health 12: 295-303, 1999.

12. Ahn YM, Yun JM, Kim HH, Seo MY, Yeom MK: Effects of dental health education on dental health knowledge and dental hygiene status in preschoolers. Child Health Nurs Res 15: 201-209, 2009.

13. Moon SJ, Park JH, Choi YC, Choi SC: The study of changes in oral health care of preschoolers in Taebaek city through oral hygiene education. J Korean Acad Pediatr Dent 36: 71-77, 2009.

14. Kang BH, Park SN, Song KY, Moon JS: Effect of a tooth-brushing education program on oral health of preschool children. J Korean Acad Nurs 38: 914-922, 2008.

15. Lee HJ, Shin SC, Cho JW, Riwe H: The case study on the effects of oral health education on primary school children. J Korean Acad Oral Health 28: 449-463, 2004.

16. Ahn SY, Hwang YS, Han SJ: Effect of oral healthcare program of exemplary kindergartens for prevent the dental caries in Incheon City, Korea. J Dent Hyg Sci 13: 501-509, 2013.

17. Ann HW, Jwa SK, Ahn SH, An YH: A comparative appraisals for oral health education throush to establishing the 
group toothbrushing facilities. Int J Clin Prev Dent 6: 35-45, 2010.

18. Kim BI: Cariview: scoring is believing. J Dent Hyg Assoc 179: 13, 2012

19. Kang SM, Jung HI, Jeong SH, Kwon HK, Kim BI: Development of a new color scale for a caries activity test. $\mathbf{J}$ Korean Acad Oral Health 34: 9-17, 2010.

20. Kim JS: A study on the mobile school dental health education model. J Korean Acad Oral Health 4: 45-60, 1980.

21. Kim JB, Ma DS, Baek DI, et al.: Preventive dentistry. 5th ed.
Komoonsa, Seoul, pp.22, 89, 283, 2009.

22. You EK, Sohn MY, Choi SC, Kim KC, Park YD, Park JH: Plaque control effect of tooth brushing instruction. J Korean Acad Pediatr Dent 39: 17-25, 2012.

23. Eom MR, Jeong DB, Park DY: Enhancement of plaque control score following individualized repeated instruction. J Korean Acad Oral Health 33: 10-18, 2009.

24. Choi YK, Park DY, Jeong DB: Relationship among adequacy, awareness of the difficulty in toothbrushing and plaque score. J Korean Acad Oral Health 33: 192-200, 2009. 